\title{
LINEAR DEGENERATE PARABOLIC EQUATIONS IN BOUNDED DOMAINS: CONTROLLABILITY AND OBSERVABILITY
}

\author{
P. Cannarsa, ${ }^{1}$ G. Fragnelli, ${ }^{1}$ and J. Vancostenoble ${ }^{2}$ \\ ${ }^{1}$ Dipartimento di Matematica, Università di Roma "Tor Vergata", via della Ricerca Scientifica, \\ 1,00133 ROMA, Italy, \{cannarsafragnell $\} @$ mat.uniroam2.it ${ }^{*}$, \\ ${ }^{2}$ Laboratoire M.I.P., U.M.R. C.N.R.S. 5640, Université Paul Sabatier Toulouse III, 118 route de \\ Narbonne, 31062 Toulouse Cedex 4, France, vancoste@mip.ups-tlse.fr
}

\begin{abstract}
In this paper we study controllability properties of linear degenerate parabolic equations. Due to degeneracy, classical null controllability results do not hold in general. Thus we investigate results of 'regional null controllability', showing that we can drive the solution to rest at time $T$ on a subset of the space domain, contained in the set where the equation is nondegenerate.
\end{abstract}

keywords: linear degenerate equations, regional null controllability, persistent regional null controllability.

\section{Introduction}

This paper is concerned with null controllability for the degenerate heat equation:

$$
\left\{\begin{array}{l}
u_{t}-\left(a(x) u_{x}\right)_{x}+b(t, x) u_{x}+c(t, x) u=h(t, x) \chi_{(\alpha, \beta)}(x), \\
u(t, 0)=u(t, 1)=0 \\
u(0, x)=u_{0}(x)
\end{array}\right.
$$

where $(t, x) \in\left(0, T^{\prime}\right) \times(0,1), u_{0} \in L^{2}(0,1), h \in L^{2}\left(\left(0, T^{\prime}\right) \times(0,1)\right)$, $0 \leq \alpha<\beta \leq 1$ and $T^{\prime}>T>0$ fixed. Moreover, assume that $b, c \in$ $L^{\infty}\left(\left(0, T^{\prime}\right) \times(0,1)\right)$ and

$$
\begin{gathered}
a:[0,1] \rightarrow[0,+\infty) \text { is } \mathcal{C}[0,1] \cap \mathcal{C}^{1}(0,1], \frac{1}{a} \in L^{1}(0,1), \\
a(0)=0 \text { and } a>0 \text { on }(0,1] .
\end{gathered}
$$

*Paper written with financial support of "Istituto Nazionale di Alta Matematica".

Please use the following format when citing this chapter:

Cannarsa, P., Fragnelli, G., and Vancostenoble, J., 2006, in IFIP International Federation for Information Processing, Volume 202, Systems, Control, Modeling and Optimization, eds. Ceragioli, F., Dontchev, A., Furuta, H., Marti, K., Pandolfi, L., (Boston: Springer), pp. 163-173. 
Note that, under suitable assumptions on $b$, the problem is well-posed in the sense of semigroup theory, working in appropriate weighted spaces.

Interest in degenerate parabolic equations as the one above is motivated by applications to probability (see, e.g., [7]) as well as to physical problems (see, e.g., [11]). Moreover, while null controllability for nondegenerate parabolic operators of second order in bounded domains has been studied in several papers (see, e.g. $[10,8]$ ), the same problem seems widely open in the case of degenerate equations.

We recall the standard notion of null controllability.

DEFINITION 1 (i): A given initial condition $u_{0} \in L^{2}(0,1)$ is null controllable in time $T>0$ if there exists $h \in L^{2}((0, T) \times(0,1))$ such that the solution $u$ of (1) satisfies $u(T) \equiv 0$ in $(0,1)$.

(ii): Equation (1) is null controllable in time $T>0$ if for all $u_{0} \in L^{2}(0,1)$ there exists $h \in L^{2}((0, T) \times(0,1))$ such that the solution $u$ of $(1)$ satisfies $u(T) \equiv 0$ in $(0,1)$.

It is well-known that null controllability in any time $T>0$ holds for equation (1) in the nondegenerate case, i.e., if $a$ is assumed to be positive on $[0,1]$ (see for instance $[10,8]$ ). On the contrary simple examples (see, e.g., [6]) show that null controllability fails due to the degeneracy of $a$.

In [6] and in [4], problem (1) is considered, under different assumptions on $a$, in the special case $b \equiv 0$ and $b \neq 0, c(t, x) u=f(t, x, u)$, respectively. In both cases the following notion of regional null controllability has been developed.

Definition 2 (Regional Null CONTRollability, [6]) Set $b \equiv 0$. Equation (1) is regional null controllable in time $T$ if for all $u_{0} \in L^{2}(0,1)$, and $\delta \in(0, \beta-\alpha)$, there exists $h \in L^{2}((0, T) \times(0,1))$ such that the solution $u$ of (1) satisfies

$$
u(T, x)=0 \text { for } x \in(\alpha+\delta, 1)
$$

The proof given in [6] to show that the solution of (1) satisfies (3) is based on an observability inequality for a suitable adjoint problem. Such an inequality is obtained by an appropriate use of cut-off functions and Carleman estimates (see, e.g., [1], [9], or [12]) for nondegenerate parabolic operators. In [4] and in the present paper the main feature of our approach is that we use a new method of proof. Indeed, instead of deducing null controllability from observability, we derive the result directly, using cut-off functions and the fact that equation of (1) is null controllable when $x$ varies in any subinterval $I \subset \subset(0,1]$, where $a$ in nondegenerate. Although, in the present paper, we have focussed our attention on linear equations, we believe that our approach can be extended to 
more general problems such as semilinear equations, higher space dimensions, and so on.

We note that global null controllability is a property stronger than (3) in the sense that it is automatically preserved with time. More precisely, if $u(T) \equiv 0$ in $(0,1)$ and if we stop controlling the system at time $T$, then for all $t \geq T$, $u(t) \equiv 0$ in $(0,1)$. On the contrary, regional null controllability is a weaker property: due to the uncontrolled part on $(0, \alpha+\delta),(3)$ is no more preserved with time if we stop controlling at time $T$. Thus, it is important to improve the previous result, as shown in [6] or [4], proving that the solution can be forced to vanish identically on $(\alpha+\delta, 1)$ during a given time interval $\left(T, T^{\prime}\right)$, i.e. that the solution is persistent regional null controllable.

Definition 3 (Persistent REgional NUll Controllability, [6]) Set $b \equiv 0$. Equation (1) is persistent regional null controllable in time $T^{\prime}>$ $T>0$ if for all $u_{0} \in L^{2}(0,1)$, and $\delta \in(0, \beta-\alpha)$, there exists $h \in$ $L^{2}\left(\left(0, T^{\prime}\right) \times(0,1)\right)$ such that the solution $u$ of $(1)$ satisfies

$$
u(t, x)=0 \text { for }(t, x) \in\left(T, T^{\prime}\right) \times(\alpha+\delta, 1) .
$$

In the present paper, we extend the above definitions and results to the case of $b \neq 0$, that is

$$
u_{t}-\left(a(x) u_{x}\right)_{x}+b(t, x) u_{x}+c(t, x) u=h(t, x) \chi_{(\alpha, \beta)}(x),
$$

where the coefficients $b$ and $c$ satisfy suitable conditions so that the problem is well-posed. In particular, the coefficient $b$ will be assumed to satisfy a bound of the form $|b(t, x)| \leq K \sqrt{a(x)}$, a condition which is well-known in the literature (see also Remark 5).

As an application of our null controllability results, we derive observability inequalities for a class of linear degenerate parabolic equations which includes the adjoint systems of certain optimal control problems considered in [6] (see Corollaries 9 and 10 ).

The paper is organized as follows: in sections 1 and 2 we discuss the wellposedness of equation (1), introducing function spaces and operators, and state our controllability results. The proofs of these results are given in section 3 .

\section{Well-posedness}

In this section we make the following assumptions: 
Assumption 4 Let $0 \leq \alpha<\beta \leq 1$ and $T^{\prime}>T>0$ be fixed. Assume that

$$
\begin{gathered}
a:[0,1] \rightarrow[0,+\infty) \text { is } \mathcal{C}[0,1] \cap \mathcal{C}^{1}(0,1], \frac{1}{a} \in L^{1}(0,1), \\
a(0)=0 \text { and } a>0 \text { on }(0,1\} ; \\
b, c \in L^{\infty}\left(\left(0, T^{\prime}\right) \times(0,1)\right) ; \\
\exists K>0 \text { such that } \mid b(t, x)\} \leq K \sqrt{a(x)} \text { for }(t, x) \in\left(0, T^{\prime}\right) \times(0,1) .
\end{gathered}
$$

Observe that (6) is, for example, satisfied by $a(x):=x^{p}, p<1$.

REMARK 5 The assumption (8), with the other assumptions, ensures that the Markov process described by the operator $C u:=-\left(a u_{x}\right)_{x}+b u_{x}$ in $[0,1]$ doesn't reach the point $x=0$, while the point $x=1$ is an absorbing barrier since $u(t, 1)=0$. This implies that, if we set the problem in $C([0,1])$ instead of $L^{2}(0,1)$, then we don't need a boundary condition at $x=0$ (see, e.g., [7]).

Let us consider the linear degenerate parabolic equation on $(0,1)$ :

$$
\left\{\begin{array}{l}
u_{t}-\left(a(x) u_{x}\right)_{x}+b(t, x) u_{x}+c(t, x) u=h(t, x) \chi_{(\alpha, \beta)}(x) \\
u(t, 0)=u(t, 1)=0 \\
u(0, x)=u_{0}(x)
\end{array}\right.
$$

where $(t, x) \in\left(0, T^{\prime}\right) \times(0,1), u_{0} \in L^{2}(0,1)$ and $h \in L^{2}\left(\left(0, T^{\prime}\right) \times(0,1)\right)$.

For well-posedness, we introduce the following weighted spaces

$$
\begin{aligned}
& H_{a}^{1}:=\left\{u \in L^{2}(0,1) \mid u \text { locally absolutely continuous in }(0,1],\right. \\
& \left.\sqrt{a} u_{x} \in L^{2}(0,1) \text { and } u(1)=u(0)=0\right\} \text {, }
\end{aligned}
$$

and

$$
H_{a}^{2}:=\left\{u \in H_{a}^{1}(0,1) \mid a u_{x} \in H^{1}(0,1)\right\},
$$

with the norms

$$
\|u\|_{H_{a}^{1}}^{2}:=\|u\|_{L^{2}(0,1)}^{2}+\left\|\sqrt{a} u_{x}\right\|_{L^{2}(0,1)}^{2}
$$

and

$$
\|u\|_{H_{a}^{2}}^{2}:=\|u\|_{H_{a}^{1}}^{2}+\left\|\left(a u_{x}\right)_{x}\right\|_{L^{2}(0,1)}^{2} .
$$

We define the operator $(A, D(A))$ by

$$
D(A)=H_{a}^{2} \text { and } \forall u \in D(A), A u:=\left(a u_{x}\right)_{x} .
$$


We recall the following properties of $(A, D(A))$ (see [2] for a proof in the case $a(0)=a(1)=0$, and [6] for the proof in our case):

Proposition 6 The operator $A: D(A) \rightarrow L^{2}(0,1)$ is a closed self-adjoint negative operator with dense domain.

Hence, $A$ is the infinitesimal generator of a strongly continuous semigroup $e^{t A}$ on $L^{2}(0,1)$. Moreover, one can show that $e^{t A}$ is analytic, even if we make no use of such a property. Since $A$ is a generator, working in the spaces considered above, we have that (9) is well-posed in the sense of semigroup theory:

THEOREM 7 Under Hypothesis 1 , for every $h \in L^{2}\left(\left(0, T^{\prime}\right) \times(0,1)\right)$ and for every $u_{0} \in L^{2}(0,1)$, there exists a unique weak solution $u$ of $(9)$ such that $u \in \mathcal{C}^{0}\left(\left[0, T^{\prime}\right] ; L^{2}(0,1)\right) \cap L^{2}\left(0, T^{\prime} ; H_{a}^{1}\right)$. Moreover, if $u_{0} \in H_{a}^{1}(0,1)$, then

$$
u \in \mathcal{U}:=H^{1}\left(0, T^{\prime} ; L^{2}(0,1)\right) \cap L^{2}\left(0, T^{\prime} ; H_{a}^{2}\right) \cap \mathcal{C}^{0}\left(\left[0, T^{\prime}\right] ; H_{a}^{1}\right),
$$

and

$$
\begin{aligned}
& \sup _{t \in\left[0, T^{\prime}\right\}}\left(\|u(t)\|_{H_{a}^{1}}^{2}\right)+\int_{0}^{T^{\prime}}\left(\left\|u_{t}\right\|_{L^{2}(0,1)}^{2}+\left\|\left(a u_{x}\right)_{x}\right\|_{L^{2}(0,1)}^{2}\right) d t \\
& \quad \leq C\left(\left\|u_{0}\right\|_{H_{a}^{1}}^{2}+\|h\|_{L^{2}\left(\left(0, T^{\prime}\right) \times(0,1)\right)}^{2},\right.
\end{aligned}
$$

where $C$ is a positive constant.

\section{Controllability results}

Assume that Assumption 41 is satisfied. Using the fact that $a$ is nondegenerate on $(\alpha, 1)$ and a classical result known for linear nondegenerate parabolic equations in bounded domains (see for example $[10,8]$ ), we will now give a direct proof of regional null controllability for the linear degenerate problem (9).

THEOREM 8 Assume Assumption 4. Then the following holds.

(i) Regional null controllability. Given $T>0, u_{0} \in L^{2}(0,1)$, and $\delta \in$ $(0, \beta-\alpha)$, there exists $h \in L^{2}((0, T) \times(0,1))$ such that the solution $u$ of $(9)$ satisfies

$$
u(T, x)=0 \text { for } x \in(\alpha+\delta, 1) .
$$

Moreover, there exists a constant $C_{T}>0$ independent of $u_{0}$ such that

$$
\int_{0}^{T} \int_{0}^{1} h^{2}(t, x) d x d t \leq C_{T} \int_{0}^{1} u_{0}^{2}(x) d x .
$$

(ii) Persistent regional null controllability. Given $T^{\prime}>T>0, u_{0} \in$ 
$L^{2}(0,1)$, and $\delta \in(0, \beta-\alpha)$, there exists $h \in L^{2}\left(\left(0, T^{\prime}\right) \times(0,1)\right)$ such that the solution $u$ of $(9)$ satisfies

$$
u(t, x)=0 \text { for }(t, x) \in\left(T, T^{\prime}\right) \times(\alpha+\delta, 1) .
$$

Moreover, there exists a constant $C_{T, T^{\prime}}>0$ such that

$$
\int_{0}^{T^{\prime}} \int_{0}^{1} h^{2}(t, x) d x d t \leq C_{T, T^{\prime}} \int_{0}^{1} u_{0}^{2}(x) d x .
$$

This result was proved in [6] and in [4] in the case $b \equiv 0$ and $b \neq 0$, respectively, and $a \in C^{1}[0,1]$. In particular, in [6], the proof was based on suitable regional observability inequalities which constituted the major technical part of the paper. Here, following [4], we give a different proof: we can deduce directly (i) from the classical null controllability results known for nondegenerate parabolic equations. Then, (ii) follows from (i) (as in [6]). Recently in [3] the null controllability result stated in the previous theorem is improved in the sense that global null controllability is proved for the following equation

$$
u_{t}-\left(a(x) u_{x}\right)_{x}+f(t, x, u)=h(t, x) \chi_{(\alpha, \beta)}(x), \quad(t, x) \in(0, T) \times(0,1) .
$$

As an application of Theorem 12.(i), we will deduce directly the regional observability inequality found in [6]. Consider the adjoint problem associated to (9)

$$
\begin{cases}\varphi_{t}+\left(a \varphi_{x}\right)_{x}+(b \varphi)_{x}-c \varphi=0, & (x, t) \in(0, T) \times(0,1) \\ \varphi(t, 0)=\varphi(t, 1)=0, & t \in(0, T)\end{cases}
$$

Then the following corollary holds.

COROLLARY 9 For all $\delta \in(0, \beta-\alpha)$ there exists a positive constant $K_{T}$ such that, for all $\varphi$ solution of $(15)$ in $\mathcal{U}$,

$$
\int_{0}^{1} \varphi^{2}(0, x) d x \leq K_{T}\left(\int_{0}^{T} \int_{\alpha}^{\beta} \varphi^{2}(t, x) d x d t+\int_{0}^{\alpha+\delta} \varphi^{2}(T, x) d x\right) .
$$

Similarly, as a consequence of the persistent regional null controllability result above one can deduce the second observability inequality given in [6] for the non homogeneous adjoint problem. Indeed, given the adjoint system

$$
\begin{cases}\varphi_{t}+\left(a \varphi_{x}\right)_{x}+(b \varphi)_{x}-c \varphi=G(t, x) \chi_{\left(T, T^{\prime}\right)}(t), & (x, t) \in\left(0, T^{\prime}\right) \times(0,1), \\ \varphi(t, 0)=\varphi(t, 1)=0, & t \in\left(0, T^{\prime}\right),\end{cases}
$$

one can prove the next result. 
Corollary 10 For all $\delta \in(0, \beta-\alpha)$ there exists a positive constant $K_{T^{\prime}}$ such that, for all $\varphi$ solution of $(17)$ in $\mathcal{U}$,

$$
\begin{aligned}
\int_{0}^{1} \varphi^{2}(0, x) d x & \leq K_{T^{\prime}}\left(\int_{0}^{T^{\prime}} \int_{\alpha}^{\beta} \varphi^{2}(t, x) d x d t\right. \\
& \left.+\int_{0}^{\alpha+\delta} \varphi^{2}\left(T^{\prime}, x\right) d x+\int_{T}^{T^{\prime}} \int_{0}^{\alpha+\delta} G^{2}(t, x) d x d t\right)
\end{aligned}
$$

\section{Proofs}

First of all, we have to observe that the well-posedness of (9) follows from the fact that $A$ generates a strongly continuous semigroup and the operator $B(t)$ defined as

$$
B(t) u:=-b(t, \cdot) u_{x}-c(t, \cdot) u
$$

can be seen as a particular perturbation of $A$ in $D\left((-A)^{\frac{1}{2}}\right)$.

\subsection{Regional null controllability}

In this section, we prove point (i) of Theorem 12. Note that (ii) follows from (i) as in [6]. We now construct cut-off functions that will be used in the following. Let $\phi \in \mathcal{C}^{\infty}([0,+\infty))$ be such that $0 \leq \phi \leq 1$, and

$$
\begin{cases}\phi(x)=0, & 0 \leq x \leq \alpha \\ \phi(x)=1, & \alpha+\delta \leq x \leq 1\end{cases}
$$

Set $\xi:=1-\phi \in \mathcal{C}^{\infty}([0,+\infty))$. Then $0 \leq \xi \leq 1$ and

$$
\begin{cases}\xi(x)=1, & 0 \leq x \leq \alpha \\ \xi(x)=0, & \alpha+\delta \leq x \leq 1\end{cases}
$$

1) Since there is no degeneracy on $(\alpha, 1)$, by classical results for linear nondegenerate parabolic equation in bounded domain (see for example [8]), we have that there exists $h_{1} \in L^{2}((0, T) \times(\alpha, 1))$ such that the solution $v$ of

$$
\left\{\begin{array}{l}
v_{t}-\left(a(x) v_{x}\right)_{x}+b(t, x) v_{x}+c(t, x) v=h_{1}(t, x) \chi_{(\alpha, \beta)}(x), \\
v(t, \alpha)=v(t, 1)=0 \\
v(0, x)=u_{0}(x)
\end{array}\right.
$$

where $(t, x) \in(0, T) \times(\alpha, 1)$, satisfies

$$
v(T, \cdot) \equiv 0 \text { on }(\alpha, 1)
$$


Moreover, there exists a constant $C>0$ such that $\left\|h_{1}\right\|_{L^{2}((0, T) \times(\alpha, 1))}^{2} \leq$ $C\left\|u_{0}\right\|_{L^{2}(\alpha, 1)}^{2}$. And so, we have:

$$
\int_{0}^{T}\|v(t)\|_{L^{2}(\alpha, 1)}^{2} d t+\int_{0}^{T}\left\|v_{x}(t)\right\|_{L^{2}(\alpha, 1)}^{2} d t \leq C_{T}\left\|u_{0}\right\|_{L^{2}(\alpha, 1)}^{2} .
$$

Then $\tilde{v}(t, x):=\phi(x) v(t, x)$ is the solution of

$$
\left\{\begin{array}{l}
\tilde{v}_{t}-\left(a(x) \tilde{v}_{x}\right)_{x}+b(t, x) \tilde{v}_{x}+c(t, x) \tilde{v}=\tilde{h}_{1}(t, x) \chi_{(\alpha, \beta)}(x), \\
\tilde{v}(t, 0)=\tilde{v}(t, 1)=0 \\
\tilde{v}(0, x)=\phi(x) u_{0}(x)
\end{array}\right.
$$

where $(t, x) \in(0, T) \times(0,1)$ and $\tilde{h}_{1}(t, x):=\phi h_{1}-\phi_{x}(a v)_{x}-\phi_{x x} a v-$ $\phi_{x} a v_{x}+b \phi_{x} v$. (Notice that $\phi_{x}, \phi_{x x}$ are supported in $(\alpha, \alpha+\delta) \subset(\alpha, \beta)$.) Clearly, $\tilde{v}$ satisfies

$$
\tilde{v}(T, \cdot) \equiv 0 \text { on }(0,1) \text {. }
$$

Moreover, using (22) below and the fact that $\tilde{h}_{1} \equiv 0$ on $(0, \alpha)$, one has

$$
\begin{aligned}
\int_{0}^{T} \int_{0}^{1} \tilde{h}_{1}^{2} d x d t & \leq K\left(\int_{0}^{T} \int_{\alpha}^{1} h_{1}^{2} d x d t+\int_{0}^{T} \int_{\alpha}^{1}|v|^{2} d x d t\right. \\
& \left.+\int_{0}^{T} \int_{\alpha}^{1}\left|v_{x}\right|^{2} d x d t\right) \\
& \leq K\left(\left\|u_{0}\right\|_{L^{2}(\alpha, 1)}^{2}+\int_{0}^{T}\|v(t)\|_{L^{2}(\alpha, 1)}^{2} d t\right. \\
& \left.+\int_{0}^{T}\left\|v_{x}\right\|_{L^{2}(\alpha, 1)}^{2} d t\right) \\
& \leq K_{T}\left\|u_{0}\right\|_{L^{2}(\alpha, 1)}^{2},
\end{aligned}
$$

where $K_{T}$ is a positive constant and depends on $T$.

3) Let $z$ be the solution of

$$
\left\{\begin{array}{l}
z_{t}-\left(a(x) z_{x}\right)_{x}+b(t, x) z_{x}+c(t, x) z=0 \\
z(t, 0)=z(t, 1)=0 \\
z(0, x)=u_{0}(x)
\end{array}\right.
$$

where $(t, x) \in(0, T) \times(0,1)$. (The well-posedness of (23) follows from Theorem 7.) Then $\tilde{z}(t, x):=\xi(x) z(t, x)$ is the solution of

$$
\left\{\begin{array}{l}
\tilde{z}_{t}-\left(a(x) \tilde{z}_{x}\right)_{x}+b(t, x) \tilde{z}_{x}+c(t, x) \tilde{z}=\tilde{h}_{2}(t, x) \chi_{(\alpha, \beta)}(x) \\
\tilde{z}(t, 0)=\tilde{z}(t, 1)=0 \\
\tilde{z}(0, x)=\psi(x) u_{0}(x)
\end{array}\right.
$$


where $(t, x) \in(0, T) \times(0,1)$ and $\tilde{h}_{2}(t, x):=-\xi_{x}(a z)_{x}-\xi_{x x} a z-\xi_{x} a z_{x}+b \xi_{x} z$ (note that $\xi_{x}, \xi_{x x}$ are supported in $(\alpha, \alpha+\delta) \subset(\alpha, \beta)$ ). Moreover, $\tilde{z}$ satisfies

$$
\tilde{z}(T, \cdot) \equiv 0 \text { on }(\alpha+\delta, 1)
$$

and, proceeding as for $\tilde{h}_{1}$, one can prove that there exists a positive constant $K_{T}$ such that

$$
\int_{0}^{T} \int_{0}^{1} \tilde{h}_{2}^{2} d x d t \leq K_{T} \int_{0}^{1} u_{0}^{2}(x) d x
$$

4) Finally, $u:=\tilde{v}+\tilde{z}$ is the solution of

$$
\left\{\begin{array}{l}
u_{t}-\left(a(x) u_{x}\right)_{x}+b(t, x) u_{x}+c(t, x) u=h(t, x) \chi_{(\alpha, \beta)}(x) \\
u(t, 0)=u(t, 1)=0 \\
u(0, x)=(\phi+\xi) u_{0}(x)=u_{0}(x)
\end{array}\right.
$$

where $(t, x) \in(0, T) \times(0,1)$ and $h:=\tilde{h}_{1}+\tilde{h}_{2}$. Moreover

$$
u(T, \cdot) \equiv 0 \text { on }(\alpha+\delta, 1)
$$

and there exists a positive constant $C_{T}$ such that

$$
\int_{0}^{T} \int_{0}^{1} h^{2} d x d t \leq C_{T} \int_{0}^{1} u_{0}^{2}(x) d x
$$

\subsection{Observability property}

In this part we prove that Theorem 12.(i) implies the observability property (16). Using (13) and (14), one directly has the next lemma.

LEMMA 11 Let $h$ be the control given by Theorem 12.(i) and u the corresponding solution of (9). Then

$$
\int_{0}^{\alpha+\delta} u^{2}(T, x) d x \leq C_{T} \int_{0}^{1} u_{0}^{2}(x) d x
$$

where $C_{T}:=e^{\left(1+K^{2}\right) T}$ and $K$ is as in Hypothesis 1 .

Proof of Corollary 9: Let $\varphi$ in $\mathcal{U}$ be a solution of (15). Let $h \in L^{2}((0, T) \times$ $(0,1))$ be the control given by Theorem 12.(i) such that

$$
\left\{\begin{array}{l}
u_{t}-\left(a(x) u_{x}\right)_{x}+b(t, x) u_{x}+c(t, x) u=h(t, x) \chi_{(\alpha, \beta)}(x) \\
u(t, 0)=u(t, 1)=0 \\
u(0, x)=\varphi(0, x) \\
u(T, x)=0, \quad x \in(\alpha+\delta, 1) .
\end{array}\right.
$$


Multiplying the previous equation by $\varphi$ and (15) by $u$, integrating over $(0,1)$ and summing up we obtain

$$
\int_{0}^{1} \frac{d}{d t}(u \varphi) d x=\int_{0}^{1} h \chi_{(\alpha, \beta)} \varphi
$$

Here we have used the fact that $|b(t, 0)| \leq K \sqrt{a(0)}=0$. Integrating over $(0, T)$ we have:

$$
\int_{0}^{1} u(T, x) \varphi(T, x) d x-\int_{0}^{1} \varphi^{2}(0, x)=\int_{0}^{T} \int_{0}^{1} h \chi_{(\alpha, \beta)} \varphi .
$$

Since $u(T, x)=0$ for all $x \in(\alpha+\delta, 1)$, one has

$$
\begin{aligned}
\int_{0}^{1} \varphi^{2}(0, x) d x & =\int_{0}^{\alpha+\delta} u(T, x) \varphi(T, x)-\int_{0}^{T} \int_{\alpha}^{\beta} h \varphi d x d t \\
& \leq \epsilon \int_{0}^{\alpha+\delta} u^{2}(T, x) d x+C_{\epsilon} \int_{0}^{\alpha+\delta} \varphi^{2}(T, x) d x \\
& +\frac{\epsilon}{2} \int_{0}^{T} \int_{\alpha}^{\beta} h^{2} d x d t+\frac{1}{2 \epsilon} \int_{0}^{T} \int_{\alpha}^{\beta} \varphi^{2} d x d t
\end{aligned}
$$

where $\epsilon>0$ will be chosen later. By Lemma 11 it follows that

$$
\begin{aligned}
\int_{0}^{1} \varphi^{2}(0, x) d x & \leq \epsilon C_{T} \int_{0}^{1} \varphi^{2}(0, x) d x+C_{\epsilon} \int_{0}^{\alpha+\delta} \varphi^{2}(T, x) d x \\
& +\epsilon \int_{0}^{T} \int_{\alpha}^{\beta} h^{2} d x d t+\frac{1}{2 \epsilon} \int_{0}^{T} \int_{\alpha}^{\beta} \varphi^{2}(t, x) d x d t .
\end{aligned}
$$

Moreover, (14) implies

$$
\begin{aligned}
\int_{0}^{1} \varphi^{2}(0, x) d x & \leq\left(\epsilon C+\epsilon C_{T}\right) \int_{0}^{1} \varphi^{2}(0, x) d x \\
& +\frac{1}{2 \epsilon} \int_{0}^{T} \int_{\alpha}^{\beta} \varphi^{2}(t, x) d x d t+C_{\epsilon} \int_{0}^{\alpha+\delta} \varphi^{2}(T, x) d x
\end{aligned}
$$

Choosing $\epsilon$ such that $1-\epsilon C-\epsilon C_{T}>0$, one has

$$
\int_{0}^{1} \varphi^{2}(0, x) d x \leq K_{\epsilon}\left(\int_{0}^{T} \int_{\alpha}^{\beta} \varphi^{2}(t, x) d x d t+\int_{0}^{\alpha+\delta} \varphi^{2}(T, x) d x\right) .
$$

\section{References}

[1] P. Albano, P. Cannarsa. Lectures on Carleman estimates for elliptic operators and applications. In preparation.

[2] M. Campiti, G. Metafune, D. Pallara. Degenerate self-adjoint evolution equations on the unit interval. Semigroup Forum 57:1-36, 1998. 
[3] P. Cannarsa, G. Fragnelli. Controllability of semilinear weakly degenerate parabolic equations. In preparation.

[4] P. Cannarsa, G. Fragnelli, J. Vancostenoble. Regional controllability of semilinear degenerate parabolic equations in bounded domains. To appear in J. Math. Anal. Appl.

[5] P. Cannarsa, P. Martinez, J. Vancostenoble. Nulle contrôlabilité régionale pour des équations de la chaleur dégénérées. Comptes Rendus Mécanique 330:397-401, 2002.

[6] P. Cannarsa, P. Martinez, J. Vancostenoble. Persistent regional controllability for a class of degenerate parabolic equations, Commun. Pure Appl. Anal. 3:607-635, 2004.

[7] K.J. Engel, R. Nagel. One-parameter Semigroups for Linear Evolution Equations, Graduate Texts in Mathematics 194, Springer-Verlag, 2000.

[8] A. V. Fursikov, O. Yu. Imanuvilov. Controllability of evolution equations. Lecture Notes Series, Research Institute of Mathematics, Global Analysis Research Center, Seoul National University 34, 1996.

[9] I. Lasiecka, R. Triggiani. Carleman estimates and exact boundary controllability for a system of coupled, non conservative second order hyperbolic equations. Partial Differential Equations Methods in Control and Shape Analysis, Lectures Notes in Pure and Applied Math. 188, Marcel Dekker, New York, 1994.

[10] G. Lebeau, L. Robbiano. Contrôle exact de l'équation de la chaleur. Comm. P.D.E. 20:335$356,1995$.

[11] P. Martinez, J. P. Raymond, J. Vancostenoble. Regional null controllability for a linearized Crocco type equation. SIAM J. Control Optim. 42:709-728, 2003.

[12] D. Tataru. Carleman estimates, unique continuation and controllability for anizotropic PDE's.Contemporary Mathematics 209:267-279, 1997. 